\title{
Quantifying contact patterns in response to COVID-19 public health measures in Canada
}

Gabrielle Brankston ${ }^{1}$, Eric Merkley², David N. Fisman, Ashleigh R. Tuite³, Zvonimir Poljak , Peter J. Loewen² and Amy L. Greer ${ }^{1,3^{*}}$

\begin{abstract}
Background: A variety of public health measures have been implemented during the COVID-19 pandemic in Canada to reduce contact between individuals. The objective of this study was to provide empirical contact pattern data to evaluate the impact of public health measures, the degree to which social contacts rebounded to normal levels, as well as direct public health efforts toward age- and location-specific settings.

Methods: Four population-based cross-sectional surveys were administered to members of a paid panel representative of Canadian adults by age, gender, official language, and region of residence during May (Survey 1), July (Survey 2), September (Survey 3), and December (Survey 4) 2020. A total of 4981 (Survey 1), 2493 (Survey 2), 2495 (Survey 3), and 2491 (Survey 4) respondents provided information about the age and setting for each direct contact made in a 24-h period. Contact matrices were constructed and contacts for those under the age of 18 years imputed. The next generation matrix approach was used to estimate the reproduction number $\left(R_{t}\right)$ for each survey. Respondents with children under 18 years estimated the number of contacts their children made in school and extracurricular settings.

Results: Estimated Rt values were 0.49 (95\% Cl: 0.29-0.69) for May, 0.48 (95\% Cl: 0.29-0.68) for July, 1.06 (95\% Cl: 0.63-1.52) for September, and $0.81(0.47-1.17)$ for December. The highest proportion of reported contacts occurred within the home (51.3\% in May), in 'other' locations (49.2\% in July) and at work (66.3 and $65.4 \%$ in September and December). Respondents with children reported an average of 22.7 (95\% Cl: 21.1-24.3) (September) and 19.0 (95\% Cl 17.7-20.4) (December) contacts at school per day per child in attendance.

Conclusion: The skewed distribution of reported contacts toward workplace settings in September and December combined with the number of reported school-related contacts suggest that these settings represent important opportunities for transmission emphasizing the need to support and ensure infection control procedures in both workplaces and schools.
\end{abstract}

Keywords: COVID-19, Contact patterns, Survey, Public health

\footnotetext{
*Correspondence: agreer@uoguelph.ca

'Department of Population Medicine, University of Guelph, Guelph, Canada

${ }^{3}$ Dalla Lana School of Public Health, University of Toronto, Toronto, Canada

Full list of author information is available at the end of the article
}

(c) The Author(s). 2021 Open Access This article is licensed under a Creative Commons Attribution 4.0 International License, which permits use, sharing, adaptation, distribution and reproduction in any medium or format, as long as you give appropriate credit to the original author(s) and the source, provide a link to the Creative Commons licence, and indicate if changes were made. The images or other third party material in this article are included in the article's Creative Commons licence, unless indicated otherwise in a credit line to the material. If material is not included in the article's Creative Commons licence and your intended use is not permitted by statutory regulation or exceeds the permitted use, you will need to obtain permission directly from the copyright holder. To view a copy of this licence, visit http://creativecommons.org/licenses/by/4.0/ The Creative Commons Public Domain Dedication waiver (http://creativecommons.org/publicdomain/zero/1.0/) applies to the data made available in this article, unless otherwise stated in a credit line to the data. 


\section{Introduction}

In March, 2020, as transmission of SARS-CoV-2, the virus that causes Coronavirus Disease 2019 (COVID-19), was increasing across Canada, provincial and local governments implemented a variety of non-pharmaceutical public health measures [1]. As a main route of transmission of SARS-CoV-2 is close contact with an infected individual [2], these restrictions were implemented to reduce contact between individuals and included a variety of physical distancing measures [1]. Canadian modelling studies have estimated that a $45-60 \%$ reduction in direct, close proximity contacts would be sufficient to suppress community transmission, reduce the number of cases of COVID-19, and protect the health care system from becoming overwhelmed [3, 4]. However, these estimates were based on model assumptions regarding expected changes in contact patterns under physical distancing measures during the COVID-19 pandemic.

For directly transmitted respiratory pathogens, transmission opportunities exist anywhere individuals can have direct close proximity contact including households, workplaces, and schools. Quantifying age-specific contact patterns improves our understanding of disease transmission, allows for the estimation of important epidemic parameters such as the reproduction number $[5,6]$, and provides empirical data for use in mathematical models which typically rely on estimates of contact patterns that have been collected in previous studies [6]. Further, quantifying changes in contact patterns at different time points during a pandemic allows us to evaluate the impact of public health measures, and identify the degree to which contacts have returned to pre-pandemic levels, as well as direct improved public health messaging efforts toward age- and locationspecific settings.

The reproduction number is defined as the average number of new cases arising from one existing case [7] and can be estimated from the growth of cases in surveillance data $[8,9]$ however, due to variations in testing and contact tracing as well as delays in reporting, case counts are not always a reliable indication of transmission. Real-time estimation of the effective reproduction number $\left(R_{t}\right)$ involves examining the ratio of change in the contact matrix from one generation of infection to the next [7]. For respiratory infections that are transmitted by direct contact, we can use survey-derived contact matrices to estimate how changes in contact patterns (where each contact represents an opportunity for transmission) influence the reproduction number $[5,6]$. If the average number of opportunities for transmission declines by a certain amount, so should the corresponding value of the reproduction number.

A number of recent studies have examined the effect of physical distancing measures on risk of SARS-CoV-2 transmission using empirically collected social contact data in China [10], Europe [11-13] and the United States [14] however, there is currently no such data reported for Canada. The objective of this study is to provide Canadian-specific data to evaluate the impact of physical distancing measures on the transmission of SARS-CoV-2. In doing so, we describe the age-specific contact patterns derived from survey data at four different time points during the COVID-19 pandemic, construct age-specific social contact matrices, and estimate the reproduction number for each time point.

\section{Methods \\ Data collection}

The study protocol was approved by the University of Guelph Research Ethics Board (protocol \#20-04-011) and the University of Toronto Research Ethics Board (protocol \#38251). The research company, Dynata (https://www.dynata.com), was contracted to conduct four cross-sectional electronic surveys in Canadians over the age of 18 years from May 7-19 (Survey 1), July 1727 (Survey 2), September 21-October 10 (Survey 3), and December 8-31 (Survey 4). No effort was made to recontact previous respondents for each subsequent survey. A quota sampling design was used to ensure the sample was representative of the Canadian population. Quotas for each survey were set for age, gender, official language and geographic region (i.e., Atlantic, Quebec, Ontario, and West) based on 2016 Canadian Census data [15]. Participants were recruited from a panel of survey respondents and paid a nominal amount for completing the survey. Panelists who logged into their Dynata account during the study period were directed to the survey if they fit the quotas being targeted. Enrollment into the survey within each stratum was on a firstcome, first-served basis. Survey responses were excluded from analysis if the survey was completed in less than one-third of the estimated completion time, if the respondent reported their age as less than 18 years, or if the survey was discontinued for exceeding the age, gender, or region quotas. Responses with duplicated entries for gender, age, postal code, date, and contact names were considered duplicate responses and removed from the dataset.

The survey instrument was adapted from the POLYMOD UK [6] and CoMix UK surveys [11]. Respondents provided information about their age, gender, province of residence, and household composition and were then asked to record all direct contacts made between 5 am on the day preceding the survey and 5 am the day of survey completion including members of their household. A direct contact was defined as anyone who was met in person and with whom a short conversation occurred, or anyone with whom the respondent had physical 
contact $[6,11]$. For each contact identified, respondents recorded the age of the contact and the setting in which the contact occurred. The survey instrument is available in the Additional file 1. As the contact diaries excluded people under the age of 18 years and with schools across the country reopening in September 2020, additional questions were added to Surveys 3 and 4 . Respondents with children under 18 were asked whether any of the children in their household had attended school, taken the school bus, attended before/after school care, or participated in extracurricular activities in the 7 days prior to survey completion. Respondents were then asked to estimate the number of contacts each of their children had in each of these settings.

Given that schools had reopened, and many people were no longer working remotely by the time Survey 3 was deployed (September, 2020), respondents were also asked to identify whether their occupation required direct contact with more than 20 people during a typical work day. Respondents in these "high contact" occupations were asked to estimate the number of people in each age category with whom they would have contact at work on a typical work day. The number of reported contacts in Surveys 3 and 4 was truncated at 75 per respondent.

\section{Data analysis}

Respondents and contacts were categorized into age groups as follows: $18-29$ years, $30-39$ years, $40-49$ years, 50-59 years, 60-69 years, and over 70 years. To ensure the sample was generally representative of the Canadian population, age, gender, region of residence, and household size of survey respondents were compared with the 2016 Canadian Census [16, 17]. Post-stratification weights were then calculated based on age and household size within Canadian region (Atlantic, Quebec, Ontario, West) using data from the 2016 Canadian census [16, 17].

The average number of contacts per respondent was calculated and stratified by age group, gender, household size, region of residence, and whether the contact diary was completed for a weekday or weekend day. The average number of contacts for each survey time period was compared to the POLYMOD UK study [6], which represents pre-pandemic contacts, by calculating the percent reduction from the mean number of contacts reported in POLYMOD.

Contact matrices were constructed for the age-specific mean number of contacts per 24-h period, adjusting for the age distribution in the Canadian population and reciprocity of contacts using the SocialMixr package in $\mathrm{R}$ [18]. The 2016 Canadian census was used to correct for the probability of contact within the population [17]. Missing contact age was sampled from other participants' contacts within the same age group.
To provide a full contact matrix with which to estimate $R_{t}$, contacts for the $0-4$ and 5-17 year age groups were imputed using a scaled version of the POLYMOD UK [6] data by multiplying the number of contacts in corresponding age groups from the POLYMOD UK [6] study by the ratio of the dominant eigenvalues of the POLYMOD UK and the observed matrices for all age groups surveyed in both studies, stratified by location of contact $[11,19]$. As schools were closed during the data collection periods in Surveys 1 and 2, school contacts were removed from the POLYMOD UK [6] data for the analysis of these two surveys only.

Proportions were calculated for those who reported that at least one of the children in their household participated in school-based or extracurricular activities. The average number of contacts was calculated per child in different settings per day (school, aftercare, bus) or per week (extracurricular) among respondents reporting participation in these activities. These estimates were not included in the contact matrices.

The next generation matrix approach was used to estimate changes in the reproduction number $\left(R_{t}\right)$ [7]. The reproduction number was estimated by multiplying $R_{0}$ prior to physical distancing interventions by the ratio of the dominant eigenvalues of the POLYMOD UK [6] and observed contact matrices under the assumptions of the social contact theory that the transmission rate is proportional to rate of social contacts $[5,20]$. A metaanalysis reported that, prior to interventions, $R_{0}$ followed a normal distribution with a mean of 2.6 and standard deviation of 0.54 [11].

To account for sampling variability and assess uncertainty, 10,000 bootstrapped samples were generated from each of the POLYMOD UK [6] and Survey 1-4 contact matrices. The ratio between the dominant eigenvalues were calculated for each bootstrapped sample of the POLYMOD UK [6] and each of the observed matrices providing a distribution of the relative change in $R_{t}$ from the observed matrices and POLYMOD UK matrices [6]. This distribution was scaled with the distribution of bootstrap samples to estimate $R_{t}$ under physical distancing measures at each of the four survey time points.

The emergence of SARS-CoV-2 variants with increased transmissibility has the potential to require more stringent public health measures [21]. To assess the theoretical impact of a more transmissible variant of SARSCoV-2, each of the scaled estimates of $R_{t}$ were multiplied by a factor of 1.56 to provide a distribution of $R_{t}$ estimates consistent with a $56 \%$ increase in transmissibility [21].

The sensitivity of the estimates of $R_{t}$ to changes in child-related contacts was assessed using previously published methods $[11,19]$. As contact diary data were collected from adults only, there is uncertainty about the 
average number of child-to-child and child-to-adult contacts under COVID-19 public health measures. To estimate the impact of varying the levels of child-related contacts on the estimates of $R_{t}$, the procedure to estimate $R_{t}$ was repeated for each Survey (1-4) with a reduction of $20,35,50,65$, and $80 \%$ of contacts from the POLYMOD UK study [6] for the 5-17 year age group.

All data were analysed using RStudio Version 1.2.5033 [22]. The code is based on the SocialMixr package [18] as well as on the work of Jarvis et al. [11]. Funding to support data collection was provided by the Public Health Agency of Canada (PHAC), The National Collaborating Centre for Infectious Diseases (NCCID), and the University of Guelph. The funders had no role in study design, data collection and analysis, decision to publish or preparation of the manuscript.

\section{Results}

We aimed to collect data from 5000 Canadians in Survey 1 (May) and 2500 Canadians in each of Surveys 2-4 (July, September, December). A total of 9120 survey responses were received for Survey 1, 4939 for Survey 2, 5310 for Survey 3, and 9599 for Survey 4. Respondents that completed the entire survey and were not screened out for any reason were included in the final sample resulting in 4981 responses for Survey 1, 2493 responses for Survey 2, 2495 responses for Survey 3, and 2491 responses for Survey 4. A summary of the exclusion process is shown in Additional file 1 Fig. A1.

The proportion of respondents living in each region, the male to female ratio, and the proportion of respondents in each age category were comparable to the 2016 Canadian Census of the population (Additional file 1 Table A1).

\section{Contact patterns}

Descriptive statistics of reported contacts are included in Additional file 1 Table A2. Data were analyzed for 11,019 reported contacts in Survey 1, 5608 in Survey 2, 12,289 in Survey 3, and 9703 in Survey 4 with an average number of $2.21,2.17,4.76$, and 3.89 contacts, respectively (Table 1 ). These represent average reductions of 79.5, 79.9, 55.9, and $64.0 \%$ for Surveys 1, 2, 3 , and 4 , in the number of contacts compared with pre-pandemic data from POLYMOD UK [6]. The reduction in contacts was consistent across age groups in Surveys 1 and 2 however, the reduction in contacts was lower in younger age groups in Survey 3 and more variable by age in Survey 4.

The age-specific contact matrices with imputed data for people younger than 18 years for each time point also reflect these reductions in the average number of contacts (Fig. 1).

\section{Estimates of $R_{t}$}

The estimated $R_{t}$ values were 0.49 (95\% Confidence Interval [CI]: 0.29-0.69) for Survey 1 (May), 0.48 (95\% CI: 0.29-0.68) for Survey 2 (July), 1.06 (95\% CI: 0.631.52) for Survey 3 (September), and $0.81(0.47-1.17)$ for Survey 4 (December) (Fig. 2). The estimated $R_{t}$ values based on a theoretical increase in transmissibility due to the emergence of a VOC as the dominant virus strain were 0.76 (95\% CI: 0.45-1.08), 0.75 (95\% CI: 0.45-1.06), 1.66 (95\% CI: $0.98-2.38$ ), and 1.26 (95\% CI: $0.74-1.82$ ) for Surveys 1-4.

The sensitivity analysis assessing the impact of reducing the number of child-related contacts by each of 20 , $35,50,65$, and $80 \%$ compared with the POLYMOD UK study [6] on estimates of $R_{t}$ resulted in estimates that were similar across the different number of assumed child-related contacts at each time point (Additional file 1 Fig. A2).

The proportion of reported contacts by setting changed over the course of the study (Table 2). Respondents in Survey 1 reported the highest proportion of their contacts to have occurred within their home $(51.3 \%)$ while those in Survey 2 reported the highest proportion of contacts to have occurred in 'other' locations such as others' homes and community places (49.2\%). Respondents in Surveys 3 and 4 reported the majority of contacts having occurred at work (66.3 and 65.4\%, respectively). School contacts were largely absent from the data in all waves of the survey because contact diaries were not collected from those under the age of 18 years and most Canadian universities had transitioned to primarily remote learning for the 2020/2021 academic year.

Figure 3 represents the average number of age-specific contacts at each time point stratified by setting in which the contact occurred. Contacts made in the respondents' home, at school (i.e., students), and in other locations are similar across the four surveys. While the average number of workplace contacts were similar in Surveys 1 and 2, reported contacts in the workplace were notably higher in Surveys 3 and 4 (Fig. 3).

A total of $688(27.6 \%)$ respondents in Survey 3 and $640(25.7 \%)$ respondents in Survey 4 reported at least one child under the age of 18 years living in their household. Of these respondents, 66.6\% (95\% CI: 62.9-70.0) (Survey 3) and 62.0\% (95\% CI 58.2-65.7) (Survey 4) reported at least one of the children in their household either participated in school-based or extracurricular activities in the 7 days prior to survey completion (Fig. 4A). Respondents reported an average of 22.7 (95\% CI: 21.124.3) (Survey 3) and 19.0 (95\% CI 17.7-20.4) (Survey 4) contacts at school per day per child in attendance. For children participating in extracurricular activities, the average number of contacts reported during these 


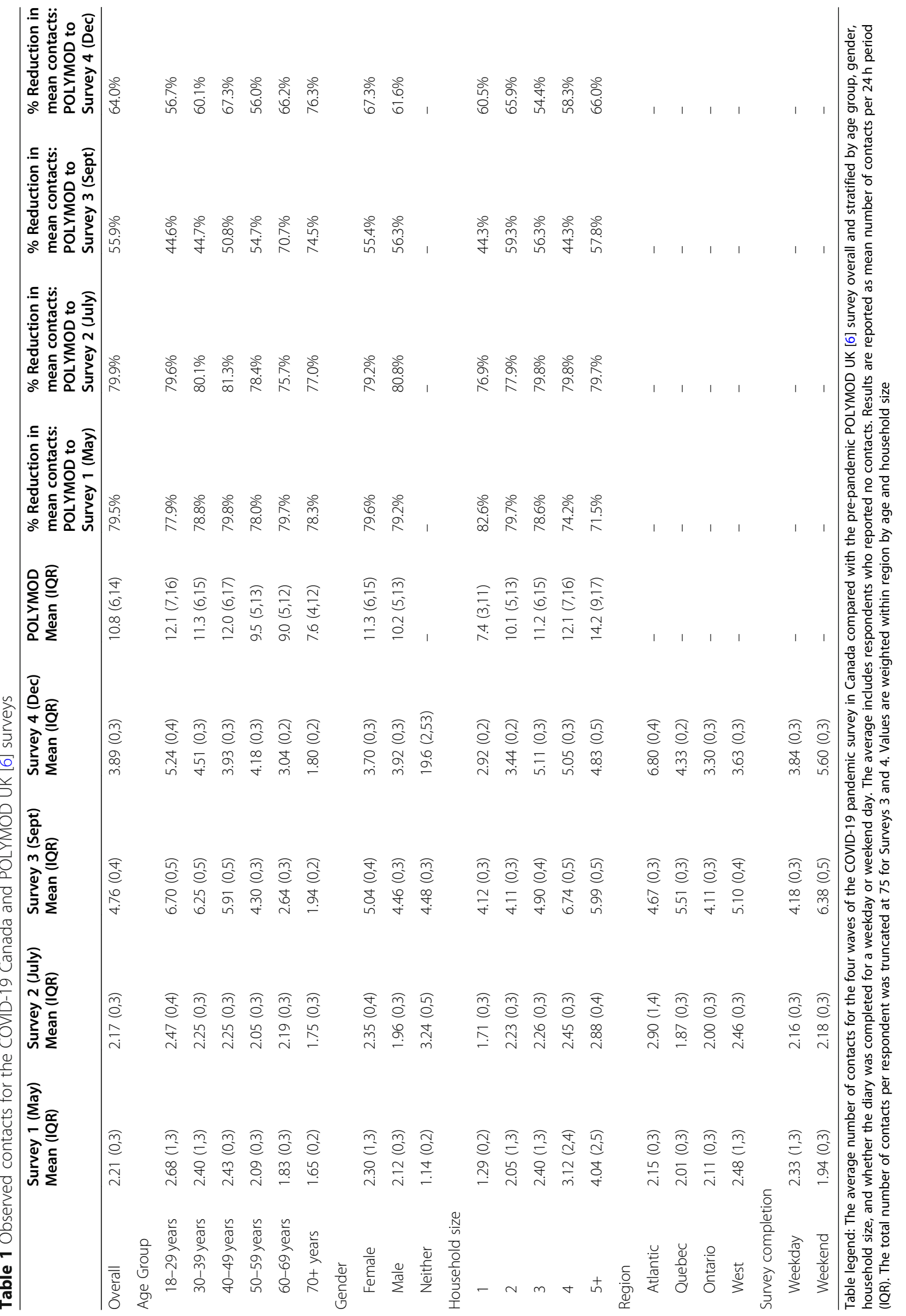




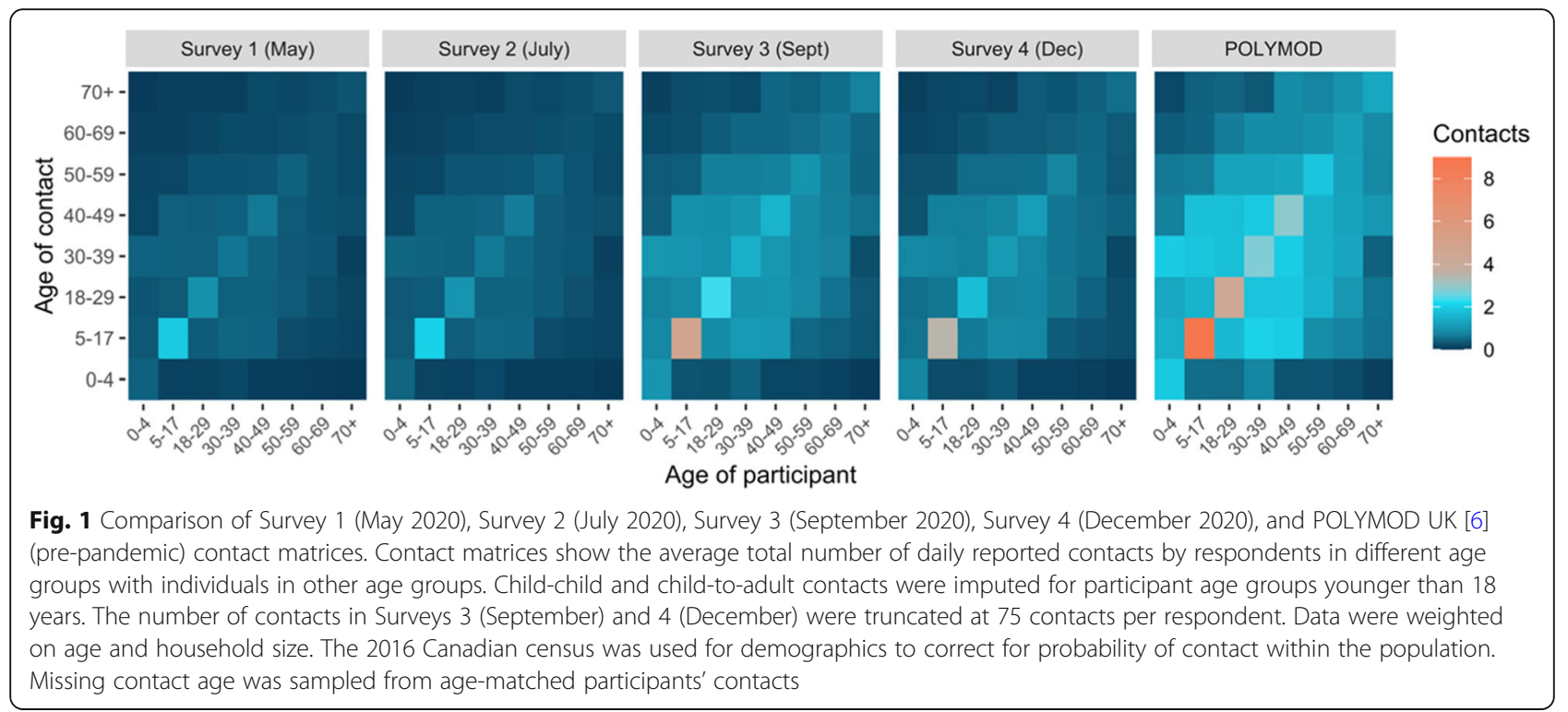

activities was 18.3 (95\% CI: 15.2-21.5) per week in Survey 3 and 11.2 (95\% CI 8.80-13.6) per week in Survey 4 (Fig. 4B).

\section{Discussion}

A summary of selected Canadian public health restrictions at each survey timepoint is included in Additional file 1 Table A3 [23]. Briefly, Canadian public health measures were most restrictive in May 2020 with continued closures of schools, universities, indoor dining and recreation. Some daycares and non-essential businesses were just beginning to reopen with limited capacity and private indoor gatherings were limited to 10 or fewer people. With the exception of schools and universities, July 2020 saw relaxing of restrictions and continued reopening of nonessential services. Elementary and secondary schools reopened for in-person learning in September 2020 and most non-essential businesses were able to reopen with some restrictions in place. By December 2020, increasing epidemic growth resulted in increasing regional restrictions and closures of non-essential businesses, indoor dining and recreation as well as a movement to remote learning for elementary and secondary students in some regions.

Consistent with the public health restrictions at each survey time point, this analysis provides evidence that, in May and July (Surveys 1 and 2), Canadians were having
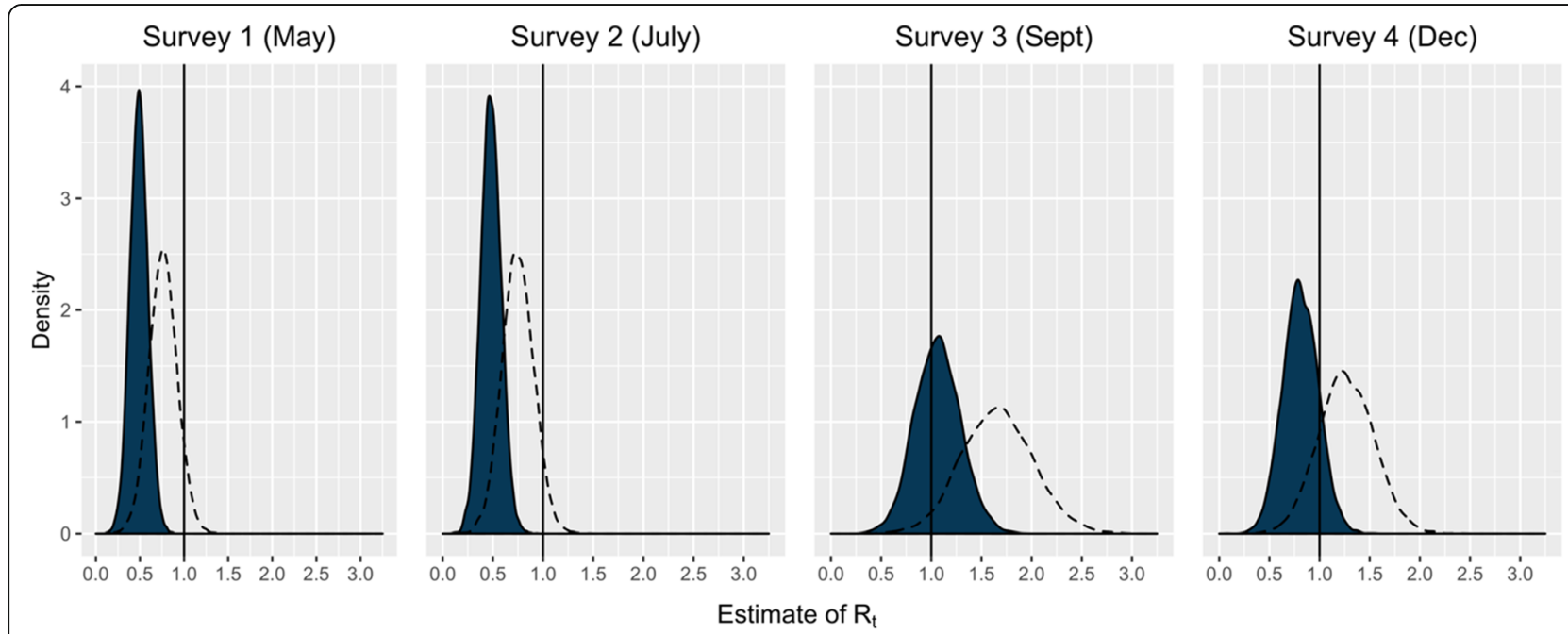

Fig. 2 Estimated distributions of $R_{t}$ for May, July, September, and December 2020 assuming a baseline normal distribution of $R_{t}$ with mean = 2.6 and $\mathrm{SD}=0.54$ prior to physical distancing measures. The dotted line represents a theoretical $56 \%$ increase in transmissibility with the variant of concern VOC 202012/01 as the dominant strain [21] 
Table 2 Proportion of reported contacts stratified by setting for each survey timepoint

\begin{tabular}{lllll}
\hline & $\begin{array}{l}\text { Survey } \mathbf{1} \text { (May) } \\
\boldsymbol{n}=\mathbf{1 1 , 0 1 9} \text { contacts }\end{array}$ & $\begin{array}{l}\text { Survey } \mathbf{2} \text { (July) } \\
\boldsymbol{n}=\mathbf{5 6 0 8} \text { contacts }\end{array}$ & $\begin{array}{l}\text { Survey } \mathbf{3} \text { (September) } \\
\boldsymbol{n = 1 2 , 2 8 9} \text { contacts }\end{array}$ & $\begin{array}{l}\text { Survey } \mathbf{4} \text { (December) } \\
\boldsymbol{n}=\mathbf{9 7 0 3} \text { contacts }\end{array}$ \\
\hline Home & $5649(51.3 \%)$ & $1951(34.8 \%)$ & $1614(13.1 \%)$ & $1384(14.3 \%)$ \\
Workplace & $1742(15.8 \%)$ & $855(15.2 \%)$ & $8145(66.3 \%)$ & $6350(65.4 \%)$ \\
School & $52(0.47 \%)$ & $42(0.75 \%)$ & $140(1.14 \%)$ & $73(0.75 \%)$ \\
Other locations & $3576(32.5 \%)$ & $2760(49.2 \%)$ & $2390(19.4 \%)$ & $1896(19.5 \%)$ \\
\hline
\end{tabular}

Table legend: The total number of contacts per respondent was truncated at 75 for survey Surveys 3 and 4 . Results are reported as number of contacts within a 24-h period (\%)

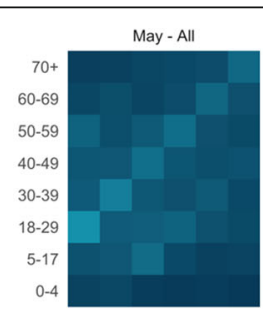

May - Home

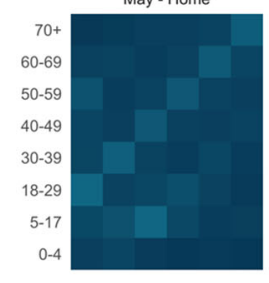

May - Work

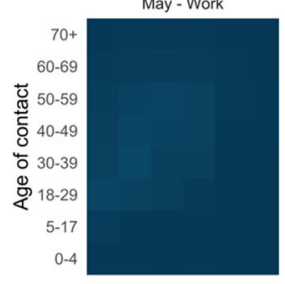

May - School

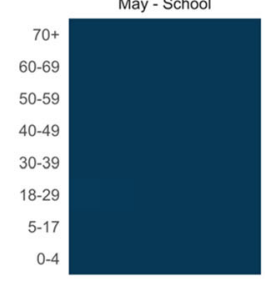

May - Other

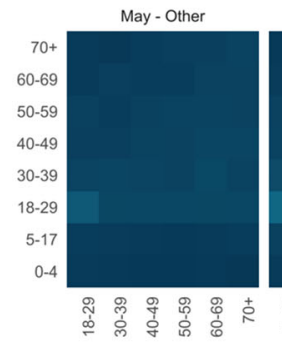

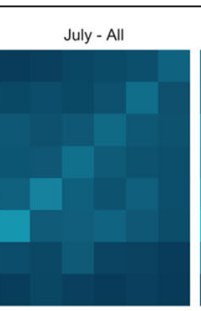

July - Home

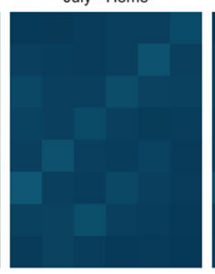

July - Work

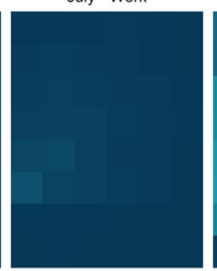

July - School

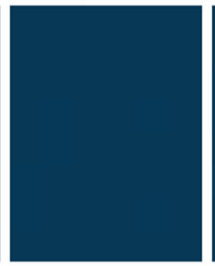

July - Other

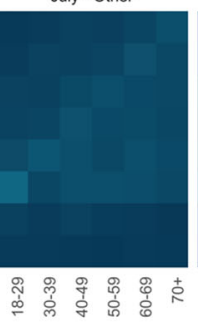

September - All

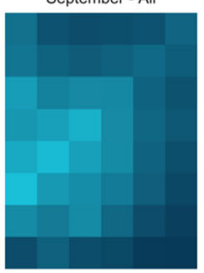

September - Home

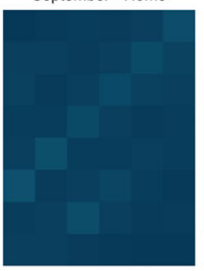

September - Work

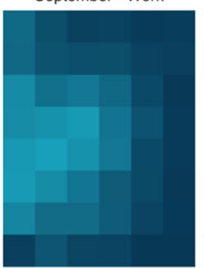

September - School

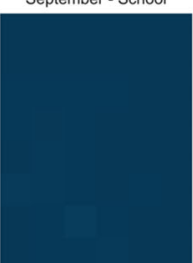

September - Other

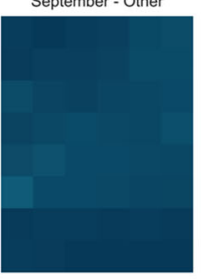

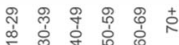
Age of participant

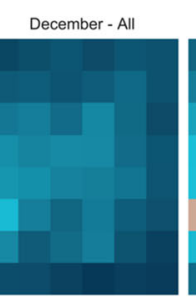

December - Home

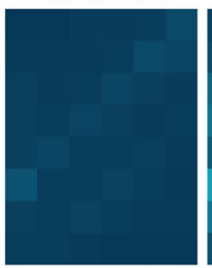

December - Work

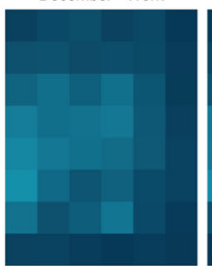

December - School

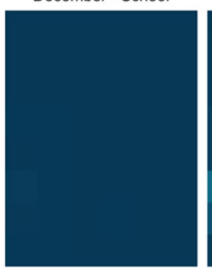

December - Other

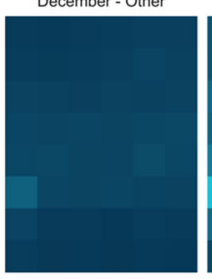

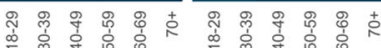

POLYMOD - All

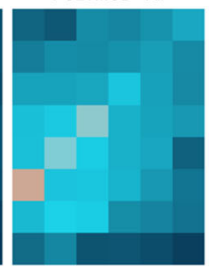

POLYMOD - Home

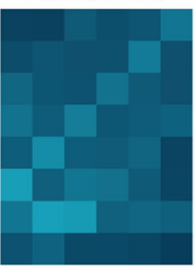

POLYMOD - Work

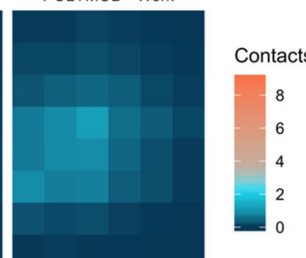

POLYMOD - School

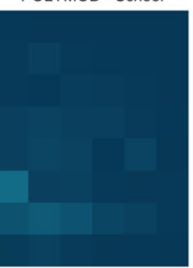

POLYMOD - Othe

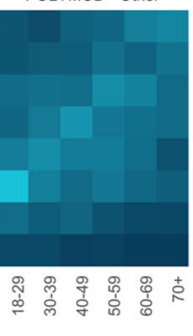

Fig. 3 Contact matrices for all reported contacts and those stratified by setting in which the contact occurred for each wave of the survey and POLYMOD UK. Age groups younger than 18 years have been removed from the POLYMOD UK matrices to facilitate comparison with the observed matrices. Settings included all contacts, contacts made at home, contacts made in the workplace, contacts made at school, and contacts made everywhere else (including social contacts). The number of contacts in the September and December surveys were truncated at 75 contacts per respondent. Data were weighted on age and household size. The 2016 Canadian census was used for demographics to correct for probability of contact within the population. Missing contact age was sampled from age-matched participants' contacts 
A

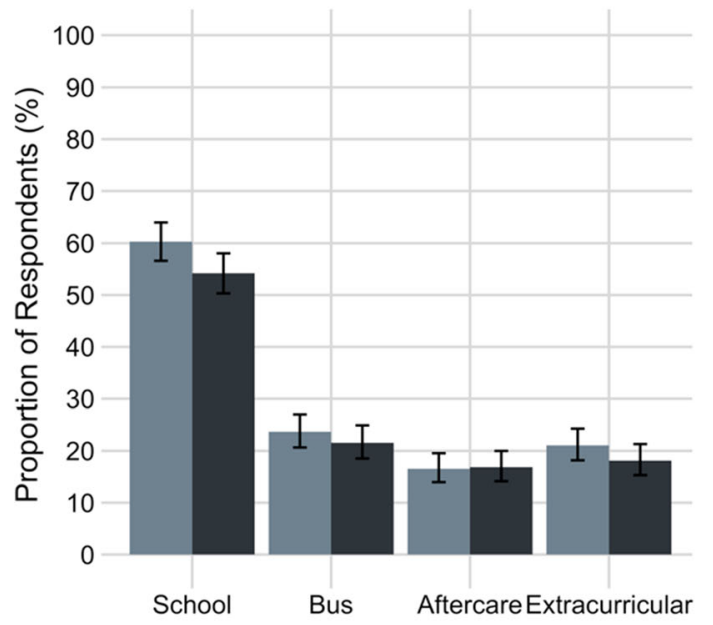

B

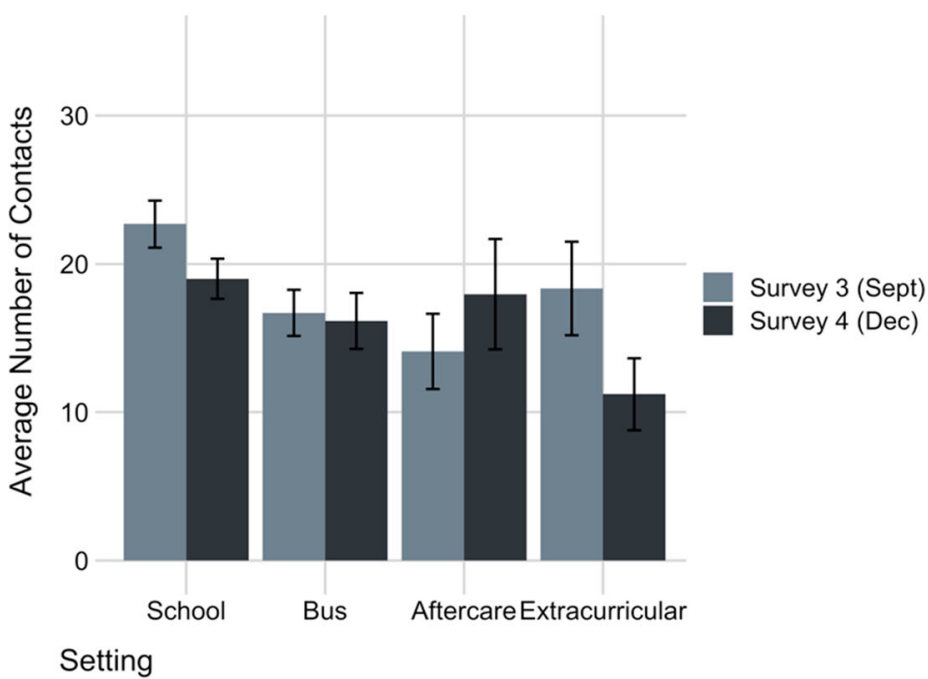

Fig. 4 A Proportion of respondents with children under the age of 18 years reporting at least one of their children attended an in-person schoolbased or extracurricular activity in the 7 days prior to survey completion. A total of 688 respondents in Survey 3 (September 2020) and 640 respondents in Survey 4 (December 2020) reported at least one child under the age of 18 years living in their household. B Estimated average number of contacts reported for children under the age of 18 years stratified by activity for respondents with household children who had reported that their children had participated in a school-based or extracurricular activity in the previous 7 days. Estimated contacts were reported per day (school, before/after school care, bus) or per week (extracurricular)

few contacts sufficient to maintain the reproduction number well below 1 , suppressing community transmission of SARS-CoV-2. The average number of contacts reported by respondents in Survey 3 (September 2020), while still more than $55 \%$ lower than those reported in the pre-pandemic POLYMOD UK study [6], had increased sufficiently to raise the reproduction number above 1 supporting a resurgence of cases of COVID-19. The contact patterns reported in Survey 4 (December 2020) likely reflect individual- and public health-level response to the epidemic growth occurring during this time period [24]. The estimated $R_{t}$ values for the May, July, December surveys were lower than reproduction numbers calculated using reported Canadian case data recognizing the incubation period of the virus and reporting delays $[24,25]$ suggesting that the POLYMOD study [6] represents an overestimate of the number of prepandemic contacts in Canada. Indeed, while there are no published pre-pandemic contact data for Canada, a synthetic pre-pandemic contact matrix for Canada suggests that POLYMOD UK overestimates Canadian prepandemic contacts in many age groups [26].

According to recent modelling studies, the reduction in the average number of contacts required to suppress transmission of SARS-CoV-2 ranged from 45 to $60 \%$ $[3,4,27]$ in comparison with study assumptions alone or with POLYMOD UK data [6], respectively. The current analysis implies epidemic growth was occurring at $55 \%$ reduction in contacts, providing empirical evidence that the reduction in contacts required to suppress a resurgence in cases is at the higher end of the spectrum of previously published estimates. Other studies of contact patterns during the COVID-19 pandemic have found that a $62-82 \%$ reduction in contacts resulted in $R_{t}$ values below $1[11,14,28]$ while others have found that $67-74 \%$ reduction in contacts resulted in $R_{t}$ values greater than $1[12,14]$. It must be noted that changes in $R_{t}$ are not solely a result of changes in contact patterns but are also influenced by factors such as travel restrictions, increased use of face masks, and increased distancing when in public places [13].

The emergence of genetic variants of SARS-CoV-2 that are more easily transmitted has the potential to impact our ability to control epidemic growth. The theoretical estimates of $R_{t}$ based on $56 \%$ higher transmissibility of the virus [21] suggest that while control measures resulting in contact patterns seen in Surveys 1 and 2 (May and July) are likely to be enough to suppress transmission, those seen in Surveys 3 and 4 (September and December) are unlikely to be sufficient to maintain $R$ below 1 should these variants become dominant in Canada.

At the time Surveys 1 and 2 were deployed in May and July 2020, public health restrictions were beginning to be lifted and non-essential businesses and workplaces were beginning to open however, schools and daycares remained closed across the country. These restrictions 
are reflected in the contact matrices and associated $R_{t}$ values in this analysis demonstrating that physical distancing was similar at the two time points. At the time of Surveys 3 and 4, daycares and schools had also reopened with the associated increase in teachers, bus drivers, and other high contact occupations returning to work. The results of our analysis are consistent with this as the higher number of contacts reported in Surveys 3 and 4 was driven by increases in contacts in the workplace rather than increases in contacts in social and other settings.

Expected increases in school contacts were not captured in this study because of the age range of respondents and the fact that many colleges and universities offered mainly remote learning opportunities at the time of the study. However, the data presented demonstrate that children are having many contacts associated with school-based and extracurricular activities. Given that large proportions of identified SARS-CoV-2 infections in children have been asymptomatic [29], these childrelated contacts may represent important and overlooked opportunities for transmission. Whereas private social gatherings often bear the weight of blame for the second wave of COVID-19 cases the findings from this study suggest that contacts made during the course of the work/school day represent important opportunities for transmission. This highlights the importance of developing and enforcing stringent infection prevention and control practices in these settings.

\section{Limitations}

The inherent risk in all surveys of being unrepresentative of the target population may have been amplified by the online nature of the survey which limited participation to those who use the Internet. Self-reporting of behaviours introduced the potential for recall and response bias. Social desirability bias carries with it the risk of underestimating the true number of total contacts as well as contacts in socially undesirable settings. These data provide no information about mitigation measures such as mask use associated with each contact. The results provide evidence that the POLYMOD UK [6] study is an imperfect representation of contact patterns in the Canadian population, however in the absence of published pre-pandemic contact data for Canada, it is a commonly used comparator. Finally, this analysis is based on contacts across Canada and does not account for any geographic variation in the number of contacts across regions potentially masking local differences.

\section{Conclusion}

In this paper, we have quantified the effect of reducing social contact numbers on the reproduction number of COVID-19. The skewed distribution of reported contacts toward workplace settings in Surveys 3 and 4 combined with the large numbers of reported schoolrelated contacts provides evidence that these settings represent important opportunities for transmission. While transmission opportunities exist in many different settings, these data emphasize the need to support and ensure evidence-based infection prevention and control procedures in both workplaces and schools.

\section{Supplementary Information}

The online version contains supplementary material available at https://doi. org/10.1186/s12889-021-12080-1.

Additional file 1.

Additional file 2 .

Acknowledgements

We thank J. Lau for support in programming the survey.

Authors' contributions

All authors (GB, EM, DF, AT, ZP, PL, and AG) contributed to the study conception and design. Survey design and data collection were performed by $G B, A G, P L$, and $E M$. Data analysis was conducted by GB. The first draft of the manuscript was written by GB and all co-authors (EM, DF, AT, ZP, PL, and $A G)$ commented on previous versions of the manuscript. GB, EM, DF, AT, ZP, $P L$, and $A G$ read and approved the final manuscript. The author(s) read and approved the final manuscript.

\section{Authors' information}

Not applicable.

\section{Funding}

GB and AG are supported by the Canada Research Chairs program. DN and AT are supported by the Canadian Institutes for Health Research (CIHR). ZP is supported by the Natural Sciences and Engineering Research Council (NSER C). EM and PL are supported by Heritage Canada and the Social Sciences and Humanities Research Council (SSHRC). Funding to support data collection was provided by the Public Health Agency of Canada (PHAC), The National Collaborating Centre for Infectious Diseases (NCCID), and the University of Guelph. The funders had no role in study design, data collection and analysis, decision to publish or preparation of the manuscript.

\section{Availability of data and materials}

The datasets used and/or analysed during the current study are available from the corresponding author on reasonable request.

\section{Declarations}

Ethics approval and consent to participate

The study protocol was approved by the University of Guelph Research Ethics Board (protocol \#20-04-011) and the University of Toronto Research Ethics Board (protocol \#38251). All methods were performed in accordance with the relevant guidelines and regulations. Informed consent was obtained prior to survey completion by providing information about the study, ensuring anonymity and confidentiality, and providing the process to withdraw from the survey. Respondents provided informed consent after reading the study information by choosing to continue to the survey questions.

Consent for publication Not applicable.

Competing interests

The authors declare that they have no competing interests. 


\section{Author details}

'Department of Population Medicine, University of Guelph, Guelph, Canada. ${ }^{2}$ Munk School of Global Affairs \& Public Policy, University of Toronto, Toronto, Canada. ${ }^{3}$ Dalla Lana School of Public Health, University of Toronto, Toronto, Canada.

Received: 17 March 2021 Accepted: 20 October 2021

Published online: 08 November 2021

\section{References}

1. Government of Canada. Community-based measures to mitigate the spread of coronavirus disease (COVID-19) in Canada. 2020 [cited 2020 Dec 10]. Available from: https://www.canada.ca/en/public-health/services/diseases/2 019-novel-coronavirus-infection/health-professionals/public-health-mea sures-mitigate-covid-19.html

2. Chief Science Officer Expert Panel on COVID-19. The Role of Bioaerosols and Indoor Ventilation in COVID-19. Ottawa; 2020. Available from: https://www. ic.gc.ca/eic/site/063.nsf/eng/h_98176.html

3. Tuite AR, Greer AL, De Keninck S. Risk for COVID-19 resurgence related to duration and effectiveness of physical distancing in Ontario, Canada. Ann Intern Med. 2020;172(1):ITC1-14. https://doi.org/10.7326/M20-2945.

4. Anderson SC, Edwards AM, Yerlanov M, Mulberry N, Stockdale JE, lyaniwura SA, et al. Quantifying the impact of COVID-19 control measures using a Bayesian model of physical distancing. PLoS Comput Biol. 2020;16(12): e1008274. https://doi.org/10.1371/journal.pcbi.1008274.

5. Wallinga J, Teunis $P$, Kretzschmar M. Using data on social contacts to estimate age-specific transmission parameters for respiratory-spread infectious agents. Am J Epidemiol. 2006;164(10):936-44. https://doi.org/10.1 093/aje/kwj317.

6. Mossong J, Hens N, Jit M, Beutels P, Auranen K, Mikolajczyk R, et al. Social contacts and mixing patterns relevant to the spread of infectious diseases. PLOS Med. 2008;5(3):e74. Available from. https://doi.org/10.1371/journal. pmed.0050074.

7. Diekmann O, Heesterbeek JAP, Roberts MG. The construction of nextgeneration matrices for compartmental epidemic models. J R Soc Interface. 2010;7(47):873-85. https://doi.org/10.1098/rsif.2009.0386.

8. Heffernan JM, Smith RJ, Wahl LM. Perspectives on the basic reproductive ratio. J R Soc Interface. 2005;2(4):281-93. https://doi.org/10.1098/rsif.2005. 0042.

9. Alessandro Annunziatio, Tommi Asikainen, Effective Reproduction Number Estimation from Data Series, EUR 30300 EN, Publications Office of the European Union, Luxembourg. 2020. https://doi.org/10.2760/036156.

10. Zhang J, Litvinova M, Liang Y, Wang Y, Wang W, Zhao S, et al. Changes in contact patterns shape the dynamics of the COVID-19 outbreak in China. Science (80- ). 2020;368(6498):1481-6.

11. Jarvis Cl, Van Zandvoort K, Gimma A, Prem K, Auzenbergs M, O'Reilly K, et al. Quantifying the impact of physical distance measures on the transmission of COVID-19 in the UK. BMC Med. 2020;18(1):124. https://doi.org/10.1186/s12 916-020-01597-8.

12. Coletti P, Wambua J, Gimma A, Willem L, Vercruysse S, Vanhoutte B, Jarvis Cl, Van Zandvoort K, Edmunds J, Beutels P, Hens N. CoMix: comparing mixing patterns in the Belgian population during and after lockdown. Sci Rep. 2020;10(1):21885. https://doi.org/10.1038/s41598-020-78540-7.

13. Del Fava E, Cimentada J, Perrotta D, Grow A, Rampazzo F, Gil-Clavel S, Zagheni E. Differential impact of physical distancing strategies on social contacts relevant for the spread of SARS-CoV-2: evidence from a crossnational online survey, March-April 2020. BMJ Open. 2021;11(10):e050651. https://doi.org/10.1136/bmjopen-2021-050651.

14. Feehan DM, Mahmud AS. Quantifying population contact patterns in the United States during the COVID-19 pandemic. Nat Commun. 2021;12(1):893. https://doi.org/10.1038/s41467-021-20990-2.

15. Statistics Canada. Age (in Single Years) and Average Age (127) and Sex (3) for the Population of Canada, Provinces and Territories, Census Divisions, Census Subdivisions and Dissemination Areas, 2016 Census - 100\% Data (table). Census. Statistics Canada Catalogue no. 98-400. Ottawa; 2016. Available from: https://www150.statcan.gc.ca/n1/en/catalogue/98-400-X201 6003

16. Statistics Canada. Census. Families, households, and marital status. Statistics (Canada catalogue no. 98-316-X2016001). Ottawa; 2016, 2017. [cited 2020 Nov 23]. Available from: https://www12.statcan.gc.ca/census-recensement/2 016/dp-pd/prof/index.cfm?Lang=E
17. Statistics Canada. 2016 Census. Age (in Single Years) and Average Age (127) and Sex (3) for the Population of Canada, Provinces and Territories, Census Metropolitan Areas and Census Agglomerations, 2016 and 2011 Censuses. (Catalogue no. 98-400-X2016001). Ottawa; 2017. [cited 2020 Nov 19]. Available from: https://www12.statcan.gc.ca/census-recensement/2016/dp$\mathrm{pd} / \mathrm{dt}$-td/Rp-eng.cfm?TABID=2\&LANG=E\&A=R\&APATH=3\&DETAIL=0\&DIM= $0 \& \mathrm{FL}=\mathrm{A} \& \mathrm{FREE}=0 \& \mathrm{GC}=10 \& \mathrm{GL}=-1 \& \mathrm{GID}=1235626 \& \mathrm{GK}=1 \& \mathrm{GRP}=1 \& \mathrm{O}=\mathrm{D} \& \mathrm{PID}=$ 109523\&PRID=10\&PTYPE $=109445 \& S=0 \& S H O W A L L=0 \& S U B=0 \& T e m p o r a l=201$ 6\&THEME $=115 \& \mathrm{VID}=0 \& \mathrm{VNAME}$

18. Funk S. socialmixr: Social Mixing Matrices for Infectious Disease Modelling. $R$ package version 0.1.6; 2020.

19. Klepac P, Kucharski AJ, Conlan AJK, Kissler S, Tang M, Fry H, et al. Contacts in context: large-scale setting-specific social mixing matrices from the BBC Pandemic project. medRxiv. 2020; Available from: http://medrxiv.org/ content/early/2020/02/19/2020.02.16.20023754.abstract.

20. Hens N, Wallinga J. Design and Analysis of Social Contact Surveys Relevant for the Spread of Infectious Diseases. In: Wiley StatsRef: Statistics Reference Online. American Cancer Society; 2019. p. 1-15. Available from: https:// onlinelibrary.wiley.com/doi/abs/10.1002/9781118445112.stat07883.

21. Davies NG, Abbott $S$, Barnard RC, Jarvis Cl, Kucharski AJ, Munday JD, Pearson CAB, Russell TW, Tully DC, Washburne AD, Wenseleers T, Gimma A, Waites W, Wong KLM, van Zandvoort K, Silverman JD; CMMID COVID-19 Working Group; COVID-19 Genomics UK (COG-UK) Consortium, Diaz-Ordaz K, Keogh R, Eggo RM, Funk S, Jit M, Atkins KE, Edmunds WJ. Estimated transmissibility and impact of SARS-CoV-2 lineage B.1.1.7 in England. Science. 2021; 372(6538):eabg3055. https://doi.org/10.1126/science.abg3055.

22. RStudio Team. RStudio: Integrated Development for R. RStudio, PBC. Boston, MA. 2020. http://www.rstudio.com/.

23. Canadian Institute for Health Information. COVID-19 Intervention Timeline in Canada — Data Tables. Ottawa; 2021

24. Public Health Agency of Canada. Update on COVID-19 in Canada: Epidemiology and Modelling. 2020. Available from: https://www.cihi.ca/en/ covid-19-intervention-timeline-in-canada.

25. Public Health Agency of Canada/National Microbiology Lab. COVID-19: PHAC modelling group report February 25, 2021. 2021.

26. Prem K, Cook AR, Jit M. Projecting social contact matrices in 152 countries using contact surveys and demographic data. PLoS Comput Biol. 2017;13(9): 1-21. Available from:. https://doi.org/10.1371/journal.pcbi.1005697.

27. Tuite AR, Fisman DN, Greer AL. Mathematical modelling of COVID-19 transmission and mitigation strategies in the population of Ontario, Canada. CMAJ. 2020;192(19):E497-E505. https://doi.org/10.1503/cmaj.200476.

28. Backer JA, Mollema L, Vos RAE, Klinkenberg D, van der Klis FRM, de Melker $\mathrm{HE}$, et al. The impact of physical distancing measures against COVID-19 transmission on contacts and mixing patterns in the Netherlands: repeated cross-sectional surveys in 2016/2017, April 2020 and June 2020. Eurosurveillance. 2021;26(8) Available from: http://medrxiv.org/content/ea rly/2020/10/16/2020.05.18.20101501.abstract.

29. King JA, Whitten TA, Bakal JA, McAlister FA. Symptoms associated with a positive result for a swab for SARS-CoV-2 infection among children in Alberta. Can Med Assoc J. 2021;193(1):E1 LP-E9 Available from: http://www. cmaj.ca/content/193/1/E1.abstract.

\section{Publisher's Note}

Springer Nature remains neutral with regard to jurisdictional claims in published maps and institutional affiliations.

Ready to submit your research? Choose BMC and benefit from:

- fast, convenient online submission

- thorough peer review by experienced researchers in your field

- rapid publication on acceptance

- support for research data, including large and complex data types

- gold Open Access which fosters wider collaboration and increased citations

- maximum visibility for your research: over $100 \mathrm{M}$ website views per year

At $\mathrm{BMC}$, research is always in progress.

Learn more biomedcentral.com/submissions 